\title{
BMJ Open Randomised, open-label, multicentre trial comparing haemodialysis plus haemoperfusion versus haemodialysis alone in adult patients with end-stage renal disease (HD/HP vs HD): study protocol
}

Wei Lu, Geng-Ru Jiang, The HD/HP versus HD trial Group

To cite: Lu W, Jiang G-R, The HD/HP versus HD trial Group. Randomised, open-label, multicentre trial comparing haemodialysis plus haemoperfusion versus haemodialysis alone in adult patients with end-stage renal disease (HD/HP vs $H D)$ : study protocol. BMJ Open 2018;8:e022169. doi:10.1136/ bmjopen-2018-022169

- Prepublication history for this paper is available online. To view these files, please visit the journal online (http://dx.doi org/10.1136/bmjopen-2018022169).

WL and G-RJ contributed equally.

Received 23 February 2018

Revised 23 April 2018

Accepted 12 June 2018

Check for updates

(C) Author(s) (or their employer(s)) 2018. Re-use permitted under CC BY-NC. No commercial re-use. See rights and permissions. Published by BMJ.

Renal Division, Department of Internal Medicine, Xin Hua Hospital affiliated to Shanghai Jiao Tong University School of Medicine, Shanghai, China

Correspondence to Dr Wei Lu;

luwei03@xinhuamed.com.cn and Dr Geng-Ru Jiang;

jianggengru@xinhuamed.com. cn

\section{ABSTRACT}

Introduction Haemodialysis (HD) is the cornerstone treatment for patients with end-stage renal disease (ESRD). However, highly protein bound or large molecular weight uremic toxins such as phenolic and indolic compounds and homocysteine, which are associated with adverse outcomes such as cardiovascular disease of patients with ESRD, are difficult to remove via HD but can be effectively eliminates by haemoperfusion (HP). The proposed trial (referred to as HD/HP vs HD below) is a randomised, open-label, multicentre trial comparing HD plus HP versus $\mathrm{HD}$ alone in adult patients with ESRD. The primary endpoint measure is all-cause mortality. Methods and analysis We plan to enrol 1364 maintenance HD patients from 11 medical centres in Shanghai. Participants will be randomised to receive HD plus HP or HD alone at a 1:1 ratio after 1-month run-in period. In both arms, patients will receive low-flux HD at a frequency of two times a week and haemodiafiltration at a frequency of once a week. In the intervention group, subjects also received HP once every 2 weeks. Followup is scheduled at $3,6,12,18$ and 24 months after randomisation, and will consist the following: routine physical examinations, standard lab panels (blood routine, liver/residual kidney functions, tests of the coagulation system, etc), dialysis adequacy (standard $\mathrm{Kt} / \mathrm{V}$ ), chest X-ray, ECG, echocardiography, heart function rating. Adverse events will be assessed according to the international conference on harmonisation guidelines. The primary outcome is 24 -month all-cause mortality. Secondary outcomes will include cardiovascular-related mortality, the occurrence of major cardiovascular events and the quality of life.

Ethics and dissemination The study protocol has been approved by the Ethical Committees of all 11 participating centres. Clinical Research Unit of Xin Hua Hospital will oversee the study. The results will be presented at national and international academic meetings, and submitted to peer-reviewed journals for publications.

Trial registration number NCT03227770; Pre-results.
Strengths and limitations of this study

- The haemodialysis (HD)/haemoperfusion (HP) versus $\mathrm{HD}$ trial is the first clinical trial that uses mortality as the primary outcome in patients with end-stage renal disease receiving HD plus HP versus $\mathrm{HD}$ alone.

- The strength of the proposed study includes relatively long follow-up (24 months), large sample size $(n=1364)$ and multiple centres representing a major metropolitan area in China.

- A major weakness of the proposed study is the estimation of uremic toxin removal efficiency using only standard tests [eg, intact parathyroid hormone (iPTH), high -sensitivity CRP (hsCRP), -macroglobulin and homocysteine]. Many relevant protein-bound uremic toxins, including phenolic and indolic compounds, will not be measured (due to practical issues).

\section{INTRODUCTION}

Despite the advance in blood purification technology and pharmacological treatment, mortality rates of patients with end-stage renal disease ESRD undergoing renal replacement treatment including haemodialysis (HD) and peritoneal dialysis remain high. Cardiovascular disease (CVD) is the leading cause of death in maintenance HD (MHD) patients. ${ }^{1}$ According to the US Renal Data System, although the mortality rate of MHD patients has dropped by $26 \%$ between 1993 and 2012, there was no significant decrease in death caused by CVD. ${ }^{2}$ In Japan, the Japanese society for dialysis therapy renal data registry reported that CVD mortality accounts for nearly $40 \%$ of all causes of mortality. ${ }^{3}$

There are emerging evidences suggesting that uremic toxins are associated with significant morbidity and mortality in patients with 
ESRD. ${ }^{4}$ Based on the physicochemical properties, the European Uremic Toxin Work Group classified uremic toxins into three major categories: (1) small, water-soluble molecules such as urea and creatinine, which can be efficiently removed by HD; (2) middle molecules larger than $500 \mathrm{Da}$ such as parathyroid hormone, 2-macroglobulin and $\mathrm{C}$ reactive protein (CRP), which can be removed by peritoneal dialysis or high-flux HD or haemofiltration and (3) protein-bound molecules such as phenolic and indolic compounds, homocysteine (Hcy) that are difficult to remove via HD or haemofiltration. ${ }^{5}$ Recent studies showed that the occurrence of death in patients with ESRD is closely associated with the low efficiency of removing middle molecule and protein-bound toxins by using the conventional dialysis therapies. ${ }^{6-12}$

Haemoperfusion (HP) allows for the removal of uremic toxins by direct contact with activated charcoal or resin via adsorption and has been one of the preferred methods to enhance poison clearance in intoxication in the clinical practice. Clinical applications of various models of extracorporeal blood purification technologies with the high-to-low clearance rates of large-molecular weight and protein-bound uremic toxins are in the following order: HP $>$ bio artificial kidney $>$ haemodiafiltration (HDF) $>$ haemofiltration $>$ HD. ${ }^{1314}$ Results from previous small-scaled trials demonstrated that HP combined with HD, conducted by adding a commercially available HP apparatus to the dialysis circuit in series with a standard haemodialyser, is an effective approach to remove small water-soluble solutes, and for middle molecule and protein-bound uremic toxins such as inflammatory cytokines and advanced glycation end products. ${ }^{13-17}$ Based on the above results, we hypothesised that HD plus HP may plausibly improve clinical outcomes of HD patients, and thus, in this HD/HP versus HD trial, we outlined the first clinical trial to observe whether HP combined with HD is superior to HD alone in the improvement of survival by significantly reducing the 24-month all-cause and cardiovascular mortality in MHD patients compared with those receiving $\mathrm{HD}$ alone.

\section{OBJECTIVES}

The primary outcome of the $\mathrm{HD} / \mathrm{HP}$ versus $\mathrm{HD}$ trial is to test if HD plus HP treatment is superior to regular HD alone in reducing all-cause mortality in MHD patients. We hypothesise that patients receiving HD plus HP treatment have lower rate of all-cause mortality than those receiving HD alone. The secondary outcome is to test if HD plus HP treatment is superior to regular HD treatment in terms of reducing cardiovascular-related mortality and major cardiovascular events (MACEs) as well as improving the quality of life.

\section{Study design}

The study will be conducted as a multicentre, openlabel, randomised controlled trial. The study will consist of a 1-month run-in period and a 24-month period of intervention and follow-up. The study flow is summarised in figure 1.

\section{Eligibility criteria}

Patients must meet all of the following criteria to be eligible:

1. Aged 18-75years.

2. Regular blood purification treatment at least 3 months before enrolled in this study.

3. Standard Kt/V $\geq 1.2$.

Subjects with one of more of the following conditions will be excluded:

1. White cell count $<4 \times 10^{9} / \mathrm{L}$ and/orplatelet count $<100 \times 10^{9} / \mathrm{L}$.

2. Cerebral haemorrhage in the past 12 weeks.

3. MACEs in the past 8 weeks.

4. Severe heart failure (New York Heart Association class III or IV). ${ }^{18}$

5. Active gastrointestinal bleeding or coagulation dysfunction.

6. Malignant tumour.

7. Active infection.

8. Pregnancy or lactation.

9. Participating in clinical trials in the past 3 months.

10. Mental disabilities.

\section{Sample size calculation}

Sample size calculation is based on the following assumptions: (1) (two sided) at 0.05 ; (2) 1 - at $80 \%$; (3) $18 \%$ 24-month all-cause mortality in the control $\operatorname{arm}^{12}$; (4) a reduction of 24-month all-cause mortality by $30 \%$ (to $12.6 \%$ ). Expecting a $15 \%$ attrition rate, a total of 682 patients per arm is needed, thus a total of 1364 patients will be needed in this HD/HP versus HD trial. There are currently around 2000 MHD patients meeting eligibility criteria in total in the 11 participating centres of this $\mathrm{HD} / \mathrm{HP}$ versus HD trial, which demonstrated that we are capable to achieving adequate participant enrolment to reach target sample size.

\section{Randomisation and treatment}

MHD patients treated in 11 centres across Shanghai, China who met the inclusion criteria will be enrolled into the study. After signing the informed consent, as shown in table 1 , in the 1-month run-in period, the baseline clinical data will be obtained and then patients will be randomised into two treatment arms: experimental group (HD plus HP treatment group) and control group (HD treatment group). Subjects will be allocated to the two arms with a 1:1 ratio using simple randomisation. Randomisation sequence will be generated by the statistics research section of the Clinical Research Unit of Xin Hua Hospital, and distributed to each of the participating centres using opaque envelops for concealment.

Patients randomised to the control group will receive low-flux HD treatment at a frequency of two times a week and online HDF) treatment at a frequency of once a week, with each treatment session lasing 4 hours. In the 


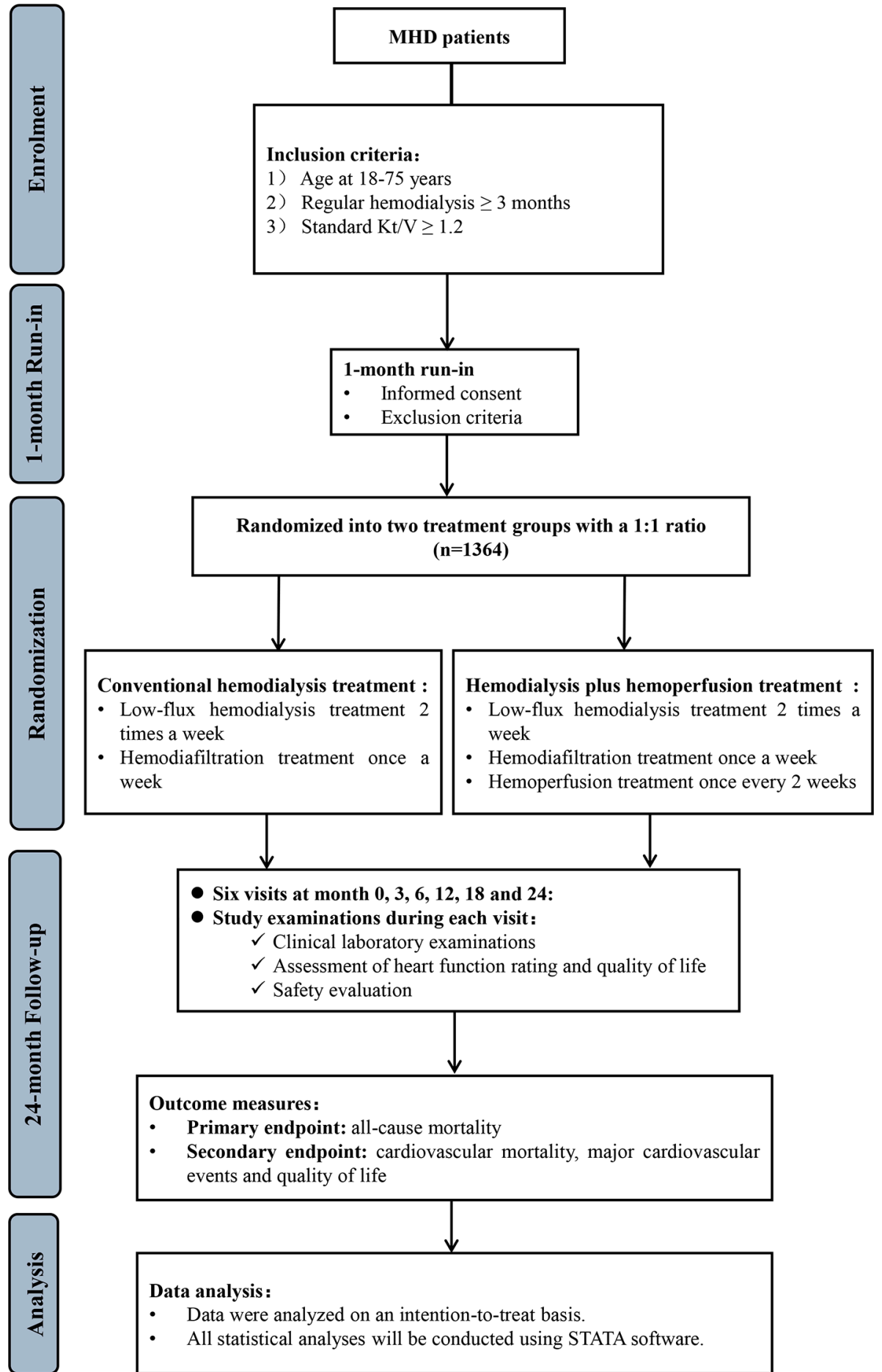

Figure 1 Study flow diagram of the HD/HP versus HD trial. HD, haemodialysis; HP, haemoperfusion; MHD, maintenance haemodialysis.

experiment group, in addition to the treatments in the control arm, HP will be conducted once every 2 weeks using a HA 130 resin HP apparatus containing $130 \mathrm{~mL}$ resin. During the treatment session, patients receiving HD and HP for the first 2 hours using a HA 130 resin HP apparatus containing $130 \mathrm{~mL}$ resin (Zhuhai Jafron Biomedical, China) and the blood flow rate maintains between 150 and $200 \mathrm{~mL} / \mathrm{min}$. After 2 hours when the HP apparatus was depleted, the HP cartridge will be removed and the blood went through the low-flux HD dialyser alone for the rest 2 hours with the blood flow rate between 200 and $250 \mathrm{~mL} / \mathrm{min}$ (figure 2). The dialysate flow rate was $500 \mathrm{~mL} / \mathrm{min}$. Heparin, weighing $0.3-0.5 \mathrm{mg} / \mathrm{kg}$ at the initial $10 \mathrm{~min}$ and $4-6 \mathrm{mg} / 30 \mathrm{~min}$ thereafter until the end of treatment session was used. Online HDF will be conducted in the postdilution mode and the volume of replacement fluid prescription will be 15-20 L. 
Table 1 Study visits of the HD/HP versus HD trial

\begin{tabular}{|c|c|c|c|c|c|c|c|}
\hline & V1 & V2 & V3 & V4 & V5 & V6 & v7 \\
\hline & $-1 M$ & OM & $3 M \pm 14 d$ & $6 M \pm 14 d$ & $12 M \pm 14 d$ & $18 M \pm 14 d$ & $\overline{24 M \pm 14 d}$ \\
\hline Consent form & $x$ & & & & & & \\
\hline Medical history & $x$ & $x$ & $x$ & $x$ & $x$ & $x$ & $x$ \\
\hline Physical examination & $x$ & $x$ & $x$ & $x$ & $x$ & $x$ & $x$ \\
\hline Eligibility & & $x$ & & & & & \\
\hline Dialysis regimen & $x$ & $x$ & $x$ & $x$ & $x$ & $x$ & $x$ \\
\hline Blood routine and coagulation test & $x$ & $x$ & $x$ & $x$ & $x$ & $x$ & $x$ \\
\hline Blood chemistry ${ }^{*}$ & $x$ & & & $x$ & $x$ & $x$ & $x$ \\
\hline Residual kidney function & $x$ & & & & & & \\
\hline Serum iPTH & $x$ & & & $x$ & $x$ & $x$ & $x$ \\
\hline Serum hsCRP & $x$ & & & $x$ & $x$ & $x$ & $x$ \\
\hline Serum $\beta_{2}$-microglobulin & $x$ & & & $x$ & $x$ & $x$ & $x$ \\
\hline Serum cysteine & $x$ & & & $x$ & $x$ & $x$ & $x$ \\
\hline Standard Kt/N & $x$ & & & $x$ & $x$ & $x$ & $x$ \\
\hline ECG & $x$ & & & & $x$ & & $x$ \\
\hline Chest X-ray & $x$ & & & & $x$ & & $x$ \\
\hline Echocardiography & $x$ & & & & $x$ & & $x$ \\
\hline Heart function rating & $x$ & $x$ & $x$ & $x$ & $x$ & $x$ & $x$ \\
\hline KDQOL-SF & & $x$ & & & $x$ & & $x$ \\
\hline Adjustment of dialysis regimen & & $x$ & $x$ & $x$ & $x$ & $x$ & $x$ \\
\hline Comorbidity & $x$ & $x$ & $x$ & $x$ & $x$ & $x$ & $x$ \\
\hline Medications & $x$ & $x$ & $x$ & $x$ & $x$ & $x$ & $x$ \\
\hline Adverse events & & $x$ & $x$ & $x$ & $x$ & $x$ & $x$ \\
\hline
\end{tabular}

*Blood chemistry includes liver function series (total bilirubin, direct bilirubin, alanine aminotransferase, aspartate aminotransferase, serum total protein, albumin, $\gamma$-glutamyltransferase and alkaline phosphatase), serum blood urea nitrogen and creatinine, electrolytes (natrium, potassium, calcium and phosphate), glucose, creatine kinase and its isoform creatine kinase - MB (CK-MB), troponin I, myohemoglobin, lactate dehydrogenase, hydroxybutyrate dehydrogenase, pro b-type natriuretic peptide.

HD, haemodialysis; HP, haemoperfusion; KDQOL-SF, Kidney Disease Quality of Life Short Form.

\section{Data collection and management}

Clinical data including medical history, physical examination, dialysis regimen, dialysis adequacy determined by standard Kt/V, ECG, chest X-ray, echocardiography, heart function rating, the quality of life assessed by the Kidney Disease Quality of Life Short Form, ${ }^{19}$ blood routines, liver function, residual kidney function ${ }^{20}$ and other biochemical index of patients will be recorded in an electronic case report form (eCRF) at a secure encrypted database by Viedoc Electronic Data Capture (EDC), which enables an audit trail and is certified by the Good Clinical Practice (GCP). An outline of the study visits and examinations to be performed is shown in table 1 .

After verification of the recorded data to source data by the data monitoring committee (DMC), recorded data in the eCRF by Viedoc EDC will be exported to a STATA file for further statistical analysis. Any missing data, data out of prelimits and possible duplication for each endpoint will be queried and internally validated by the DMC before locking the database. The DMC is independent of the study investigators and has no competing interests. After the completion of the study, the study database will be locked and data are archived for 10 years in accordance with local policy. Records will be kept on the occurrence of adverse events (AEs), time and cause of patients missing the interview, newly onset or recurrent cardiovascular events and the

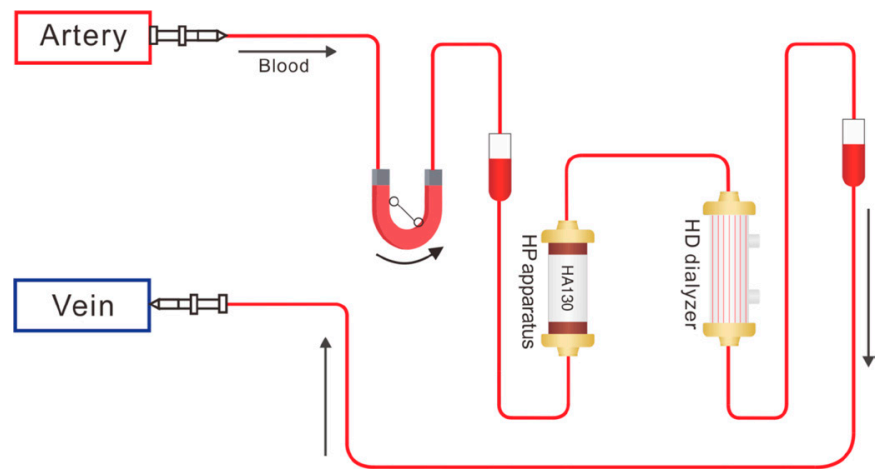

Figure 2 A schematic diagram of haemodialysis (HD) plus haemoperfusion (HP) treatment. 
patients' death. An interim analysis will be performed on primary endpoint when half of the patients have been randomised and have completed the 24-month follow-up. The interim analysis will be performed by the Clinical Research Unit of Xin Hua Hospital, who has no competing interests, and will decide whether to continue with the trial.

\section{AE monitoring}

The AEs are categorised according to the international conference on harmonisation guidelines. ${ }^{21} 22$ The causal relationship to study drug is determined by the physician. All AEs will be collected and recorded after the patient has provided consent and is included in the trial. An $\mathrm{AE}$ which meets the criteria for a serious $\mathrm{AE}$ (SAE) between study enrolment and hospital discharge will be reported to the DMC and trial management committee within 24 hours. An SAE report should be completed for any event where doubt exists regarding its status of seriousness. All SAEs should be followed to resolution or stabilisation. Non-SAEs should be followed to resolution or stabilisation, or reported as SAEs if they become serious. Follow-up is also required for non-SAEs that cause interruption or discontinuation of study drug. If patients suffer harm as a result of their participation in the trial, they will receive free treatment and sufficient compensation.

\section{Data analysis}

For data analysis, categorical variables will be analysed using the 2 or Fisher's exact test. Continuous variables will be analysed using Student's t-test on normal distribution or the Mann-Whitney U test otherwise. Primary outcomes will be analysed using the Kaplan-Meier method followed by the log-rank test. Multivariate Cox regression will be used to analysed factors that could influence all-cause mortality and CVD mortality after the adjustment for multiple relevant traditional and uraemia-related risk factors for mortality. Data were analysed on an intentionto-treat basis. All statistical analyses will be conducted using STATA V.14.0.

\section{Patient and public involvement}

The study participants were not involved beyond the standard roles as the subjects of the proposed trial. The public was not involved.

\section{Ethics and dissemination}

Substantive protocol amendments will be reported, reviewed and approved by the local medical ethical committee before application. The study may be subject to inspection and audit by Clinical Research Unit of Xin Hua Hospital to ensure adherence to the guidelines of GCP. The frequency of monitoring visits will be determined by the site enrolment rate. On study completion, the study monitor will conduct a study termination visit.
Results of this study will be presented at national and international scientific meetings, and publications will be submitted to peer-reviewed journals.

\section{Consent and confidentiality}

This trial will be performed in accordance with the Declaration of Helsinki. Each patient will need to agree with a fully informed consent form and sign it. All laboratory specimens, data collection, reports, administrative forms and the process itself will be identified by a coded ID to maintain patient confidentiality. All records that include the name or personal identifier will be stored separately from records identified by ID. Datasets on the website will be protected by password. The principal investigators will need to sign agreements to maintain the confidentiality of all patients. Study information relating to any patient will not be released externally without the written permission of the patient.

\section{DISCUSSION}

There are several unique features of the HD/HP versus HD trial compared with prior trials of combined HP and HD. The HD/HP versus HD trial is the first clinical trial that uses mortality as the primary outcome in patients with ESRD receiving HD plus HP treatment versus receiving HD treatment alone. As HD combined with HP is an effective approach to remove small water-soluble solutes, and for middle molecule and protein-bound uremic toxins, it may plausibly improve patient prognosis, thus, we hypothesised that the combination treatment of HD plus HP might reduce all-cause mortality of MHD patients. Second, the strengths of the proposed study include relatively long follow-up (24 months), large sample size $(n=1364)$ and multiple centres representing a major metropolitan area in China.

The design of this study has the limitation that we only select several representative middle molecules and protein-bound uremic toxins such as iPTH, hsCRP, 2-macroglobulin and Hcy and tested the removal efficiency of these uremic toxins by both treatment arms. Testing for protein-bound uremic toxins such as phenolic and indolic compounds are unavailable in the clinical setting, which is one main limitation of this study. Additionally, HP treatment will have potential complications and side effects such as transient leucocytopaenia and thrombocytopaenia, minor reduction in fibrinogen and fibronectin and blood reactions to biocompatible materials. ${ }^{23}$ Thus, in the experiment group, in addition to the HD treatments in the control arm, HP will be added once every 2 weeks. Third, due to the higher cost (both HD-related equipment and accessories) associated with high-flux HD, low-flux HD two times weekly plus HDF once weekly is a widely used protocol across China. Thus, the results of the proposed trial must be interpreted with caution when 
extrapolating to USA and Europe, where the conventional protocol is high-flux HD three times per week. Regardless, the results of the proposed trial will provide useful information that could potentially change the medical practice in the USA and Europe if additional trials are conducted in subjects receiving high-flux HD three times per week.

In summary, the HD/HP versus HD trial will present an opportunity to assessing the efficacy of HD plus HP in improving the survival of MHD patients. Results from this trial may provide guidance to the optimisation of blood purification therapy in MHD patients.

Acknowledgements The authors thank the study participants, trial staff and investigators for their participation. Principal investigators at the clinical sites are listed below according to the number of patients in each site: Geng-Ru Jiang from XinHua Hospital affiliated to Shanghai Jiao Tong University School of Medicine; Xiao-Qiang Ding from ZhongShan Hospital affiliated to Fudan University; Zhao-Hui Ni from Renji Hospital affiliated to Shanghai Jiao Tong University School of Medicine; Xiao-Nong Chen from Rui Jin Hospital affiliated to Shanghai Jiao Tong University School of Medicine; Wei-Jie Yuan from Shanghai First People's Hospital affiliated of Shanghai Jiao Tong University; Nian-Song Wang from Shanghai Sixth People's Hospital affiliated to Shanghai Jiao Tong University; Zhi-Yong Guo from ChangHai Hospital affiliated to Second Military Medical University; Feng Ding from Shanghai Ninth People's Hospital affiliated to Shanghai Jiao Tong University School of Medicine; Yue-Yi Deng from Long Hua Hospital affiliated to Shanghai University of Traditional Chinese Medicine; Chen Yu from Shanghai Tongji Hospital affiliated to Tongji University School of Medicine; Rong Zhou from Yangpu Hospital affiliated to Tongji University School of Medicine.

Collaborators Geng-Ru Jiang from Xin Hua Hospital affiliated to Shanghai Jiao Tong University School of Medicine; Xiao-Qiang Ding from Zhong Shan Hospital affiliated to Fudan University; Zhao-Hui Ni from Renji Hospital affiliated to Shanghai Jiao Tong University School of Medicine; Xiao-Nong Chen from Rui Jin Hospital affiliated to Shanghai Jiao Tong University School of Medicine; Wei-Jie Yuan from Shanghai First People's Hospital affiliated of Shanghai Jiao Tong University; Nian-Song Wang from Shanghai Sixth People's Hospital affiliated to Shanghai Jiao Tong University; Zhi-Yong Guo from Chang Hai Hospital affiliated to Second Military Medical University; Feng Ding from Shanghai Ninth People's Hospital affiliated to Shanghai Jiao Tong University School of Medicine; Yue-Yi Deng from Long Hua Hospital affiliated to Shanghai University of Traditional Chinese Medicine; Chen Yu from Shanghai Tongji Hospital affiliated to Tongji University School of Medicine; Rong Zhou from Yangpu Hospital affiliated to Tongji University School of Medicine.

Contributors WL and G-RJ involved in conception and trial design and in drafting of the article. The HD/HP versus HD trial group participated in the trial. Ten other centres (Zhong Shan Hospital affiliated to Fudan University, Renji Hospital affiliated to Shanghai Jiao Tong University School of Medicine; Rui Jin Hospital affiliated to Shanghai Jiao Tong University School of Medicine; Shanghai First People's Hospital affiliated to Shanghai Jiao Tong University; Shanghai Sixth People's Hospital affiliated to Shanghai Jiao Tong University; Chang Hai Hospital affiliated to Second Military Medical University; Shanghai Ninth People's Hospital affiliated to Shanghai Jiao Tong University School of Medicine; Long Hua Hospital affiliated to Shanghai University of Traditional Chinese Medicine; Shanghai Tongji Hospital affiliated to Tongji University School of Medicine; Yangpu Hospital affiliated to Tongji University School of Medicine) participated in the trial.

Funding The HD/HP versus HD trial is being funded by Shanghai Hospital Development Center (grant No. 16CR1021A).

Competing interests None declared.

Patient consent Not required.

Ethics approval The protocol has been approved by Ethical Committee of 11 participating centres and has been assigned the following protocol ID: NCT03227770.

Provenance and peer review Not commissioned; externally peer reviewed.

Open access This is an open access article distributed in accordance with the Creative Commons Attribution Non Commercial (CC BY-NC 4.0) license, which permits others to distribute, remix, adapt, build upon this work non-commercially, and license their derivative works on different terms, provided the original work is properly cited, appropriate credit is given, any changes made indicated, and the use is non-commercial. See: http://creativecommons.org/licenses/by-nc/4.0/.

\section{REFERENCES}

1. Matsushita K, van der Velde M, Astor BC, et al. Association of estimated glomerular filtration rate and albuminuria with all-cause and cardiovascular mortality in general population cohorts: a collaborative meta-analysis. Lancet 2010;375:2073-81.

2. U.S. Renal Data System. USRDS 2017 Annual Data Report.

3. Hanafusa N, Nakai S, Iseki K, et al. Japanese society for dialysis therapy renal data registry-a window through which we can view the details of Japanese dialysis population. Kidney Int Suppl 2015:5:15-22.

4. Moradi H, Sica DA, Kalantar-Zadeh K. Cardiovascular burden associated with uremic toxins in patients with chronic kidney disease. Am J Nephrol 2013;38:136-48.

5. Vanholder R, De Smet R, Glorieux G, et al. Review on uremic toxins: classification, concentration, and interindividual variability. Kidney Int 2003;63:1934-43.

6. Vanholder R, Van Laecke S, Glorieux G. What is new in uremic toxicity? Pediatr Nephrol 2008;23:1211-21.

7. Lesaffer G, De Smet R, Lameire N, et al. Intradialytic removal of protein-bound uraemic toxins: role of solute characteristics and of dialyser membrane. Nephrol Dial Transplant 2000;15:50-7.

8. London GM, Marty C, Marchais SJ, et al. Arterial calcifications and bone histomorphometry in end-stage renal disease. J Am Soc Nephrol 2004;15:1943-51

9. Furuya R, Kumagai H, Miyata $\mathrm{T}$, et al. High plasma pentosidine level is accompanied with cardiovascular events in hemodialysis patients. Clin Exp Nephrol 2012;16:421-6.

10. Vanholder R, Schepers E, Pletinck A, et al. An update on proteinbound uremic retention solutes. J Ren Nutr 2012;22:90-4.

11. Bammens B, Evenepoel P, Verbeke K, et al. Removal of the proteinbound solute $\mathrm{p}$-cresol by convective transport: a randomized crossover study. Am J Kidney Dis 2004;44:278-85.

12. Mandolfo S, Borlandelli S, Imbasciati E. Leptin and beta2microglobulin kinetics with three different dialysis modalities. Int $\mathrm{J}$ Artif Organs 2006;29:949-55.

13. Chen SJ, Jiang GR, Shan JP, et al. Combination of maintenance hemodialysis with hemoperfusion: a safe and effective model of artificial kidney. Int J Artif Organs 2011;34:339-47.

14. Li WH, Yin YM, Chen $\mathrm{H}$, et al. Curative effect of neutral macroporous resin hemoperfusion on treating hemodialysis patients with refractory uremic pruritus. Medicine 2017;96:e6160.

15. Raine A, Cordonnier D, Ritz E. Effect of hematodialysis plus hemoperfusion on insulin resistance and nutritional status of patients with end-stage diabetic nephropathy. $J$ Int Transl Med 2015;51:180-4.

16. Zhang J, Yuan Y, An X, et al. Comparison of combined blood purification techniques in treatment of dialysis patients with uraemic pruritus. Int J Clin Exp Med 2016;9:8563-8.

17. Zhang Y, Mei CL, Rong S, et al. Effect of the combination of hemodialysis and hemoperfusion on clearing advanced glycation end products: a prospective, randomized, two-stage crossover trial in patients under maintenance hemodialysis. Blood Purif 2015;40:127-32.

18. The Criteria Committee of the New York Heart Association. Nomenclature and criteria for diagnosis of diseases of the heart and blood vessels. Boston: Little Brown, 1964.

19. Hays RD, Kallich JD, Mapes DL, et al. Kidney Disease Quality of Life Short Form (KDQOL-SF), Version 1.3: a manual for use and scoring. Santa Monica, CA: RAND, 1997.

20. Obi Y, Rhee CM, Mathew AT, et al. Residual kidney function decline and mortality in incident hemodialysis patients. J Am Soc Nephrol 2016;27:3758-68.

21. International Conference on Harmonisation. Clinical safety data management: definitions and standards for expedited reporting E2A: International Conference on Harmonisation, 1994.

22. International Conference on Harmonisation. Post-approval safety data management: definitions and standards for expedited reporting E2D: International Conference on Harmonisation, 2003.

23. Ghannoum M, Bouchard J, Nolin TD, et al. Hemoperfusion for the treatment of poisoning: technology, determinants of poison clearance, and application in clinical practice. Semin Dial 2014;27:350-61. 